\title{
Escala para nominar aptitudes sobresalientes. Validez de contenido por jueces expertos y no expertos ${ }^{1}$
}

Fabiola Zacatelco Ramírez, ${ }^{2}$ Blanca Chávez Soto² y Aurora González Granados²

\section{Introducción}

La identificación y atención de niños y adolescentes con aptitudes sobresalientes en nuestro país ha atravesado múltiples dificultades debido a imprecisiones en torno a los conceptos, criterios e indicadores involucrados con los procesos de evaluación e intervención. De ahí la importancia de realizar investigaciones y plantear nuevas alternativas para apoyar el óptimo desarrollo del sobresaliente.

Un primer paso en este sentido tiene lugar en relación con el proceso de identificación de esta población que debe darse en condiciones de equidad y en consideración con los múltiples y variados factores contextuales que inciden en su desarrollo. Por ello se requiere contar con instrumentos que, además de valorar los indicadores con mayor aceptación entre los especialistas en el área, reconozcan características determinadas por el contexto

$1 \quad$ Este trabajo fue apoyado por el proyecto PAPIIT IN304713.

2 Facultad de Estudios Superiores Zaragoza, UNAM. 
y cumplan con los requisitos para garantizar su rigor teórico y metodológico.

Al respecto, múltiples autores como Ferrando, Prieto, Ferrándiz y Sánchez (2005), Valadez, Pérez y Beltrán (2010), Zacatelco, Chávez, Lemus, Tapia y Ortiz (2010) y Zavala (2004), señalaron la importancia de contar con la opinión de los docentes, quienes a partir de la convivencia cotidiana con niños y adolescentes, pueden observar y dar cuenta de sus intereses, inquietudes, habilidades y necesidades educativas, aspectos todos importantes para el proceso de identificación de aptitudes sobresalientes. $Y$ aunque existen quienes desestiman dichas aportaciones, por considerarlas poco precisas o sesgadas en favor del rendimiento académico (Blanco, 2001), otros autores como Valadez, Meda y Zambrano (2006) han argumentado que estas limitaciones pueden ser solucionadas al ofrecer al profesor información pertinente respecto a los indicadores por observar.

Con el fin de conjuntar la experiencia del profesor con los criterios establecidos por especialistas en el área, en investigaciones previas se utilizó un instrumento propuesto en México por Covarrubias (2001), denominado "Lista para padres de familia y maestros regulares, para identificar el potencial sobresaliente en niños y niñas que cursan la educación primaria", que consta de 25 preguntas dicotómicas, con reactivos en español que incluyen criterios reportados en la literatura especializada y pueden responderse con facilidad y rapidez. No obstante, un análisis posterior permitió encontrar en él términos ambiguos como "sentido del humor especial" o "alto nivel de comprensión", además de criterios escasamente relacionados con las aptitudes sobresalientes y preguntas que demandan del profesor conocer el transcurso del desarrollo normal, para compararlo con el del evaluado.

El presente trabajo ofrece evidencias de validez de contenido para la "Escala de Nominación del Maestro", diseñada para identificar niños con aptitudes sobresalientes de educación primaria, con base en los factores propuestos por Renzulli (1979 y 2011): capacidad superior, creatividad y compromiso con la tarea, además de aspectos socioafectivos que Chan (2010), Mue- 
Iler (2009), Schick y Phillipson (2009) y Speirs y Finch (2006) relacionaron con esta población. El estudio se dividió en dos fases.

\section{Método}

Fase 1

\section{Objetivo}

Seleccionar los mejores reactivos con base en el juicio de expertos.

\section{Participantes}

Cuatro doctores en Psicología con reconocida experiencia en la identificación de aptitudes sobresalientes, investigación y elaboración de instrumentos de evaluación.

\section{Herramientas}

Sesenta reactivos redactados a partir de los indicadores de aptitudes sobresalientes, 15 para cada una de las cuatro dimensiones por evaluar.

\section{Procedimiento}

Para la elaboración de los reactivos se partió de una versión previa de la escala (Zacatelco, Chávez y González, 2013), que fue utilizada para valorar a 670 niños de tercero a quinto de primaria, donde se observó que $74 \%$ de los nominados por el profesor eran confirmados como sobresalientes, a través de escalas de inteligencia, compromiso con la tarea, creatividad y autoconcepto académico. Esta versión alcanzó un índice de confiabilidad de 0.934 , a partir del Alpha de Cronbach, pero no contaba con evidencias de validez.

Con el fin de realizar un análisis de contenido a partir de jueces expertos, se esclarecieron las definiciones de las categorías por evaluar, se amplió el número de reactivos, clasificaron, organizaron al azar y enviaron a cuatro jueces expertas para 
que opinaran respecto a su ubicación en categorías y fraseo, y decidieran cuáles debían modificarse o eliminarse.

\section{Resultados}

Se eliminaron 28 reactivos y se hicieron cambios en la redacción de tres, utilizando como norma para conservarlos que contaran con al menos tres opiniones a favor. Algunos ejemplos de estos procedimientos fueron los siguientes.

- La afirmación "recuerda con detalle la información de temas escolares" fue clasificada por los cuatro jueces en la dimensión "capacidad superior" y no tuvo observaciones, por lo que permaneció sin cambios, mientras que el reactivo "los aprendizajes de una materia puede aplicarlos en otras", que tres jueces clasificaron en el mismo rubro, fue eliminado porque se manifestaron dudas en relación con su pertenencia al dominio.

- En la aseveración que indaga si el alumno "solicita información adicional a la explicada por el maestro", tres de los jueces acordaron ubicarla en "compromiso con la tarea", pero uno de ellos propuso cambios en la redacción. La versión modificada dice "busca información adicional a la explicada por el maestro".

- El enunciado "muestra curiosidad por situaciones nuevas", que se suponía relacionado con creatividad, fue ubicado en diferentes categorías por cada uno de los jueces, por lo que se decidió eliminarlo.

- El reactivo "reconoce abiertamente sus emociones" fue clasificado por los cuatro jueces expertos como parte de la dimensión "aspectos socioafectivos" y quedó sin cambios.

Con base en lo anterior, la segunda versión de la escala quedó conformada por un total de 32 reactivos. Asimismo, se cambió el formato dicotómico por uno Likert de 5 puntos para ofrecer una mayor gama de posibilidades de respuesta y contar con un instrumento más sensible. 
Fase 2

\section{Objetivo}

Recabar opiniones de profesores de primaria, en relación con los reactivos, instrucciones y formatos que conforman la escala.

\section{Participantes}

Siete profesores que se encontraban activos que impartían clases en diferentes grados de primaria.

\section{Herramientas}

- Los 32 reactivos seleccionados en la fase anterior, ocho por área.

- Cuestionario con 12 preguntas dicotómicas para que los profesores emitieran sus opiniones respecto a la integración de la escala.

\section{Procedimiento}

Se informó a los profesores el objetivo del estudio y se solicitó su participación voluntaria para responder a cinco ejemplares de la escala y posteriormente que emitieran sus opiniones en el cuestionario.

\section{Resultados}

Todos los participantes reportaron haber leído con cuidado las instrucciones y alrededor de $80 \%$ consideraron que éstas eran claras y suficientes.

En relación con el fraseo de los reactivos, 100\% afirmó que era claro y utilizaba un lenguaje de fácil comprensión. Cerca de $90 \%$ señaló que el número de reactivos era adecuado y que contaban con la información necesaria para responder.

Todos coincidieron en que los temas eran suficientes y calificaron positivamente el formato para dar sus respuestas. Ninguno opinó que algún reactivo generara incomodidad y $86 \%$ confirmó la utilidad de la escala para nominar alumnos con aptitudes sobresalientes. 


\section{Discusión}

Las imprecisiones en la conceptualización de la categoría pueden dar lugar a problemas en la identificación y atención de niños con aptitudes sobresalientes y, con ello, suscitar la pérdida o disminución de su potencial. De Zubiría (2006) y Moska (2004) indicaron que las aptitudes de estos niños no son constantes y precisan de la organización de experiencias educativas que favorezcan su desarrollo, a fin de evitar que puedan aminorarse o perderse, por lo que la generación de instrumentos como el que aquí se presenta constituyen una contribución importante.

La polémica respecto al papel del profesor en este proceso entre quienes opinan que tiene un papel privilegiado por las horas que pasa en interacción con los alumnos (Zeynep y Bayindir, 2009), y quienes dudan de su objetividad y señalan que tiende a sobrevalorar el desempeño académico (Elices y Palazuelo, 2006), hace resaltar la importancia del diseño de instrumentos que le proporcionen información relacionada con los indicadores de la aptitud sobresaliente para que pueda participar y contribuir en la identificación.

Al respecto, se pueden señalar los prometedores resultados obtenidos en la aplicación de la primera versión de esta escala, a partir de la cual 24 profesores evaluaron a 670 niños de tercero a quinto grado, con un nivel de $74 \%$ de coincidencia en la identificación con otras pruebas utilizadas (Test de matrices progresivas de Raven, Escala de compromiso con la tarea, Prueba de pensamiento creativo de Torrance y Escala de autoconcepto académico).

Con base en estos resultados, se considera que los esfuerzos por mejorar este instrumento y reunir evidencias a favor de su validez de contenido implicarán una aportación en dos sentidos: en la generación de herramientas que contribuyan para la identificación de niños con aptitudes sobresalientes, y en la atención a la comunidad, a partir de la evaluación e intervención de necesidades educativas relacionadas con esta categoría, lo que es particularmente relevante para quienes viven en zonas económicamente desfavorecidas, donde la carencia o 
escasez de recursos con frecuencia limitan sus oportunidades de desarrollo.

Finalmente, es pertinente reconocer que los avances logrados hasta el momento con esta escala resultan relevantes pero no suficientes, pues aún es preciso hacer otros procedimientos que permitan obtener evidencias relacionadas con distintas formas de validez, además de realizar los análisis estadísticos que den cuenta de su consistencia interna y otras formas de confiabilidad.

\section{Referencias}

Blanco, M.C. (2001). Guía para la identificación y seguimiento de los alumnos superdotados. Barcelona: Praxis.

Chan, D. (2010). Perfectionism among Chinese Gifted and Nongifted Students in Hong Kong: The Use of the Revised Almost Perfect Scale. Journal for the Education of the Gifted, 34(1), 68-98.

Covarrubias, P. (2001). Características cognitivas y socioafectivas de los niños y niñas sobresalientes de la zona norte de México. (Tesis de maestría inédita). Universidad Autónoma de Tlaxcala. México.

De Zubiría, J. (2006). Teorías contemporáneas de la inteligencia y la excepcionalidad. Colombia: Cooperativa Editorial Magisterio.

Elices, S.J. y Palazuelo, M.M. (2006). El profesor identificador de necesidades educativas asociadas a las altas capacidades. Faisca, 11(13), 23-47.

Ferrando, M., Prieto, M.D. Ferrándiz, C. y Sánchez, C. (2005). Inteligencia y creatividad. Revista Electrónica de Investigación Psicoeducativa, 7(3), 21-50.

Moska, E.L. (2004). Identificación de los niños cas. Educar Revista de Educación, 29, 17-34. Recuperado de http://portalsej.jalisco.gob.mx.

Mueller, C.E. (2009). Protective Factors as Barriers to Depression in Gifted and Nongifted Adolescents. Gifted Child Quarterly, 53(1), 3-14. 
Renzulli, J. (1979). The Enrichment Triad Model: A Guide for Developing Defensible Programs for the Gifted, Mansfield Center. EuA: Creative Learning Press.

Renzulli, J. (2011). What Makes Giftedness? Reexamining a Definition. Kappan, 92(8), 81-89.

Schick, H. y Phillipson, S. (2009). Learning Motivation and Performance Excellence in Adolescents with High Intellectual Potential: What Really Matters? High Ability Studies, 20(1), 15-37.

Speirs, N.K. y Finch, H. (2006). Perfectionism in High-Ability Students: Relational Precursor and Influences on Achievement Motivation. Gifted Child Quarterly, 50(3), 237-251.

Valadez, D., Meda, R.M. y Zambrano G.R. (2006). Identificación de niños sobresalientes que estudian en escuelas públicas. Revista de Educación y Desarrollo, 5 (julio-septiembre), 39-45.

Valadez, D., Pérez, L. y Beltrán, J. (2010). La inteligencia emocional de los adolescentes talentosos. Faisca, 15(17), 2-17.

Zacatelco, F., Chávez, B. y González, A. (julio, 2013). Análisis psicométrico de una escala de nominación del maestro para identificar aptitudes sobresalientes: resultados preliminares. Presentado en el XXXIV Congreso Interamericano de Psicología "Por la integración de las Américas". Brasilia, Brasil.

Zacatelco, F., Chávez, B., Lemus, A., Tapia, E. y Ortiz, G. (2010). Detección de alumnos con capacidad sobresaliente en una escuela primaria urbano-marginal. Revista Mexicana de Psicología, 407-408, número especial ISSN: 0185607-3.

Zavala. B.M.A. (2004). La detección de alumnos CAS-Superdotados en las escuelas primarias. (Tesis doctoral, inédita). Universidad Autónoma de Aguascalientes, Aguascalientes, México.

Zeynep, I.H. y Bayindir, N. (2009). Awareness Level of Teachers About the Characteristics of Gifted Childrens. Australian Journal of Basic and Applied Sciences, 3(3), 2519-2527. 